Check for updates

Cite this: RSC Adv., 2017, 7, 42491

Received 19th June 2017

Accepted 25th August 2017

DOI: $10.1039 / c 7 r a 06845 j$

rsc.li/rsc-advances

\section{Crystallization and melting behavior of i-PP: a perspective from Flory's thermodynamic equilibrium theory and DSC experiment}

\author{
Abdullah K. Ahmed, ${ }^{a}$ Muhammad Atiqullah, ${ }^{\star b}$ Dev R. Pradhan ${ }^{c}$ and Mamdouh A. Al- \\ Harthi*a
}

Crystallization and melting are integral parts of the isotactic polypropylene (i-PP) end-product fabrication process. Therefore, the nonisothermal crystallization kinetics and melting behavior of an un-nucleated commercial i-PP have been pursued by integrating a new nonisothermal crystallization model, Flory's thermodynamic equilibrium theory, Gibbs-Thompson equation, and DSC experiments. By applying this simple conceptual integration, the relative crystallinity $\alpha$, temperature-dependent instantaneous crystallinity $\chi$, the crystallization kinetic triplet, and the lamellar thickness distribution have been duly correlated, as appropriate, to the level of undercooling $\theta$, crystal surface free energy $D$, and critical stable crystallite sequence number $n *$. Consequently, new insightful results, interpretations, and explanations have been concluded. The nonisothermal primary and secondary crystallizations of i-PP occur isokinetically with constant (temperature-, entropy-, and cooling rate-invariant) apparent kinetic energy $E_{a}$, which is also unaffected by $\theta, D$, and the lamella-inclusive pendant $-\mathrm{CH}_{3}$ group of the propylene repeat unit. The crystal dimension $n_{\mathrm{d}}$ varies, irrespective of $\theta$ and $D$, from cylinder to sphere, depending on the system entropic disorder. Instantaneous (athermal/heterogeneous) and sporadic (thermal/ homogeneous) nucleation processes may co-prevail. The occurrence of lamellar thickening with increasing melting temperature has been discussed, considering the chain sliding diffusion theory proposed by Hikosaka et al., and the variation of $\theta$ and $D$. The present approach also applies to the evaluation of the influence of catalyst structure, backbone defect types, and their distribution on the crystallization and melt behavior of i-PP.

\section{Introduction}

Isotactic polypropylene (i-PP) is a polymorphic semi-crystalline large-volume low-price thermoplastic. It has several unique features such as high toughness, elasticity, transparency, and impermeability; ease of processability; excellent water and chemical resistance; and balanced physical and chemical properties. Therefore, it is gaining high importance in the polyolefin business, end-use, and application. Its major applications relate to the automobile industry, food packaging, and the manufacture of toys and furniture. ${ }^{1-6}$

The fabrication of i-PP end-products involves heating and cooling which occur during processing. It melts and crystallizes upon heating and cooling, respectively. These phenomena affect the polymer structure which controls the ultimate properties listed above. The crystallization can be isothermal as well

${ }^{a}$ Department of Chemical Engineering, King Fahd University of Petroleum \& Minerals, Dhahran 31261, Saudi Arabia.E-mail: mamdouh@kfupm.edu.sa

${ }^{b}$ Center for Refining \& Petrochemicals, Research Institute, King Fahd University of Petroleum \& Minerals, Dhahran 31261, Saudi Arabia. E-mail: matiq@kfupm.edu.sa ${ }^{c}$ Analytical Laboratory, SABIC Technical Center, Riyadh 11551, Saudi Arabia as nonisothermal. However, practical processes such as injection molding, extrusion molding, extrusion blow molding, and vacuum forming mostly undergo non-isothermal crystallization. Hence, it is very important to investigate i-PP nonisothermal crystallization, particularly the kinetics, and melting behavior to generate knowledge that can be used to efficiently operate industrial fabrication processes and manufacture endproducts with better properties and improved performance. ${ }^{\mathbf{1 - 6}}$

A review of the literature on overall polypropylene thermal behavior shows the following. The published nonisothermal crystallization studies relate to i-PP with and without nucleating agents $^{7-10}$ and i-PP/inorganic filler micro- and nanocomposites. ${ }^{11-14}$ Here, the crystallization kinetics was investigated using Caze-Chuah, Jeziorny, Ozawa, Mo, and Seo-Kim models (as appropriate). ${ }^{7-14}$ However, the following can be commented regarding these models. They are not mechanistic; they are empirical; and they lack fundamental and phenomenological formalism. The model parameters do not always have adequate physical significance. Some of them do not represent the entire crystallization profile, and some experience double logarithm insensitivity and linearization problems. See ref. 15 for the details. 
We particularly summarize the study by Alamo et al. ${ }^{\mathbf{1 6}}$ who investigated, among several subjects, the effects of chainwalking defects on i-PP \% crystallinity and melting point, considering Flory's equilibrium theory. These regio-defects, resulting from 3, 1 enchainment, were introduced into the polymer backbone by polymerizing propylene using selected nickel $\alpha$-diimine precatalysts and methylaluminoxane (MAO) cocatalyst. They lowered the above thermal properties with reference to the corresponding Flory's equilibrium values. However, these authors did not investigate the influence of i-PP regio-defects on nonisothermal crystallization kinetics, which is an important subject.

We observe that the aforementioned i-PP nonisothermal crystallization studies and the report by Alamo et al. ${ }^{16}$ did not study the i-PP dynamic melting behavior and crystallization from Flory's equilibrium theory perspective, particularly considering the following three temperature-dependent dimensionless factors-level of undercooling $\theta$, crystal surface free energy $D$, and critical stable crystallite sequence number $n$ * Our focused objective is the following. Can the integration of the fundamental mechanistic crystallization model (which we published in 2013), ${ }^{15}$ Flory's thermodynamic equilibrium theory. ${ }^{16-18}$ Gibbs-Thompson equation, ${ }^{19-23}$ and DSC experiments generate new and/or better insightful results, interpretations, and explanations regarding nonisothermal i-PP crystallization and melt process? Note that such an integrated conceptual framework to study the overall polyolefin thermal behavior, as far as we know, has not been yet published. Therefore, we undertake this study. The merits of our above crystallization model over the other literature models are well documented..$^{1524-26}$ This is based on mechanistic consideration. It applies to the entire DSC nonisothermal crystallization curve. The model-predicted kinetic parameters-apparent kinetic energy $E_{\mathrm{a}}$, Avrami exponent $n$, and crystallization frequency factor $k_{0}$-have physical significance.

We plan to pursue the above objective by correlating the relative crystallinity $\alpha$, temperature-dependent instantaneous crystallinity $\chi, E_{\mathrm{a}}$, crystal dimension $n_{\mathrm{d}}$, nucleation mode $n_{\mathrm{c}}, k_{0}$, and the lamellar thickness and melting temperature, as appropriate, to $\theta, D$, and $n^{*}$. The DSC experiments, as a function of heating/cooling rates, will be conducted using an alphaphase commercial Ziegler-Natta i-PP without adding a nucleating agent.

\section{Experimental}

\subsection{Materials}

The pristine un-nucleated isotactic polypropylene (PP H1030) used in this study was a gift from National Industrialization Company (Tasnee), Saudi Arabia. As per the Tasnee data sheet, it has a melt flow rate (MFR) of $3 \mathrm{~g} / 10 \mathrm{~min}$ at $230{ }^{\circ} \mathrm{C}$ and $2.16 \mathrm{~kg}$ load; and a density of $0.900 \mathrm{~g} \mathrm{~cm}^{-3}$.

\subsection{Molecular weight and polydispersity index}

The experimental i-PP was characterized in terms of weight average molecular weight $\left(M_{\mathrm{w}}\right)$ and polydispersity index (PDI), using a Viscotek multidetector high temperature GPC (HT GPC Module 350A) instrument. The column temperature was set at $140{ }^{\circ} \mathrm{C}$. The polypropylene sample (about $21.50 \mathrm{mg}$ ), taken in a $40 \mathrm{~mL}$ glass vial, was dissolved in $10.0 \mathrm{~mL}$ butylated hydroxy toluene BHT-stabilized-1,2,4 trichlorobenzene (TCB) as follows. The vial was closed with a Teflon cap. Then the sample was completely dissolved by placing it into the Vortex Auto Sampler for $3 \mathrm{~h}$ at $140{ }^{\circ} \mathrm{C}$ under mild stirring.

Before injecting a sample, all the detectors-refractive index (RI) detector, and low angle and right angle light scattering (LALS and RALS) detectors - and the inlet pressure (IP), and the differential pressure (DP) cells were purged for $3 \mathrm{~h}$ using TCB to obtain a stable baseline. The flow rate of TCB was $1.0 \mathrm{~mL} \mathrm{~min}{ }^{-1}$. The experimental i-PP sample, having a concentration of $2.15 \mathrm{mg} \mathrm{mL}^{-1}$, was injected into the system. The run time was $60 \mathrm{~min}$. Viscotek OmniSEC software acquired the response data, generated by the LALS and RALS detectors, and calculated the weight average molecular weight $M_{\mathrm{w}}$ and the PDI, which are reported in Table 1 . The measured $M_{\mathrm{w}}$ is comparable with the viscosity average molecular weight $M_{\mathrm{v}}$ of an equivalent MFR i-PP reported in the literature. ${ }^{27}$

\subsection{Thermal property, density, and amorphous volume fraction}

The thermal properties of the experimental i-PP were measured in terms of peak melting $\left(T_{\mathrm{pm}}\right)$ and crystallization $\left(T_{\mathrm{pc}}\right)$ temperatures, and \% crystallinity $\left(X_{\mathrm{c}}\right)$ using a differential scanning calorimeter (DSC Q2000, Texas Instrument). The instrument was calibrated using indium. The three-cycle (heating-cooling-heating) experimental procedure reported in the literature was followed. ${ }^{24,28,29}$ About $5.50 \mathrm{mg}$ i-PP flake sample was taken in an aluminum pan and was tightly crimped with a lid. A similar empty pan was used as a reference. The following heating/cooling rates-5.0, 10.0, 12.5, 15.0, and $20.0{ }^{\circ} \mathrm{C}$ min-were used under nitrogen flow. After keeping the sample and reference pans in the DSC instrument, Cycle 1 was completed as follows to remove thermal history and recrystallization. The sample was first heated from room temperature to $200.0{ }^{\circ} \mathrm{C}$ at a selected heating rate; then it was kept at this temperature for $5 \mathrm{~min}$. In Cycle 2, it was cooled to room temperature at the same ramp. Finally, in Cycle 3, the sample was heated to $200.0{ }^{\circ} \mathrm{C}$, also using the same heating rate. This means that Cycles 1 to 3 experienced the same heating or cooling rate in a typical DSC run.

The data were acquired for each cycle and handled using the TA explorer software. The \% crystallinity was determined using Cycle 3 DSC output and the following relation: $\chi_{\mathrm{c}}=\left(\Delta H_{\mathrm{f}} / \Delta H_{\mathrm{f}}^{\circ}\right) \times 100$, where $\Delta H_{\mathrm{f}}$ and $\Delta H_{\mathrm{f}}^{\circ}\left(207 \mathrm{~J} \mathrm{~g}^{-1}\right)^{\mathbf{1 6 , 3 0 , 3 1}}$ are the heats of fusion of the experimental sample and the perfect (defect-free) i-PP crystal (of infinite lamellar thickness and molar mass), respectively. The measured $\chi_{\mathrm{c}}$ was subsequently used to calculate the material density $\rho_{\text {polym }}$, following the rule of additivity of volumes of polypropylene amorphous and crystalline phases: ${ }^{32} \chi_{\mathrm{c}}=\left(1 / \rho_{\text {polym }}-1 / \rho_{\mathrm{a}}\right) /\left(1 / \rho_{\mathrm{c}}-1 / \rho_{\mathrm{a}}\right)$; where $\rho=$ density; $\mathrm{a}=$ amorphous phase; $\mathrm{c}=$ crystalline phase; and polym $=$ polymer. For polypropylene, $\rho_{\mathrm{c}}=0.950 \mathrm{~g} \mathrm{~mL}^{-1}$ and $\rho_{\mathrm{a}}=$ 
Table 1 Summary of the experimental i-PP properties ${ }^{d}$

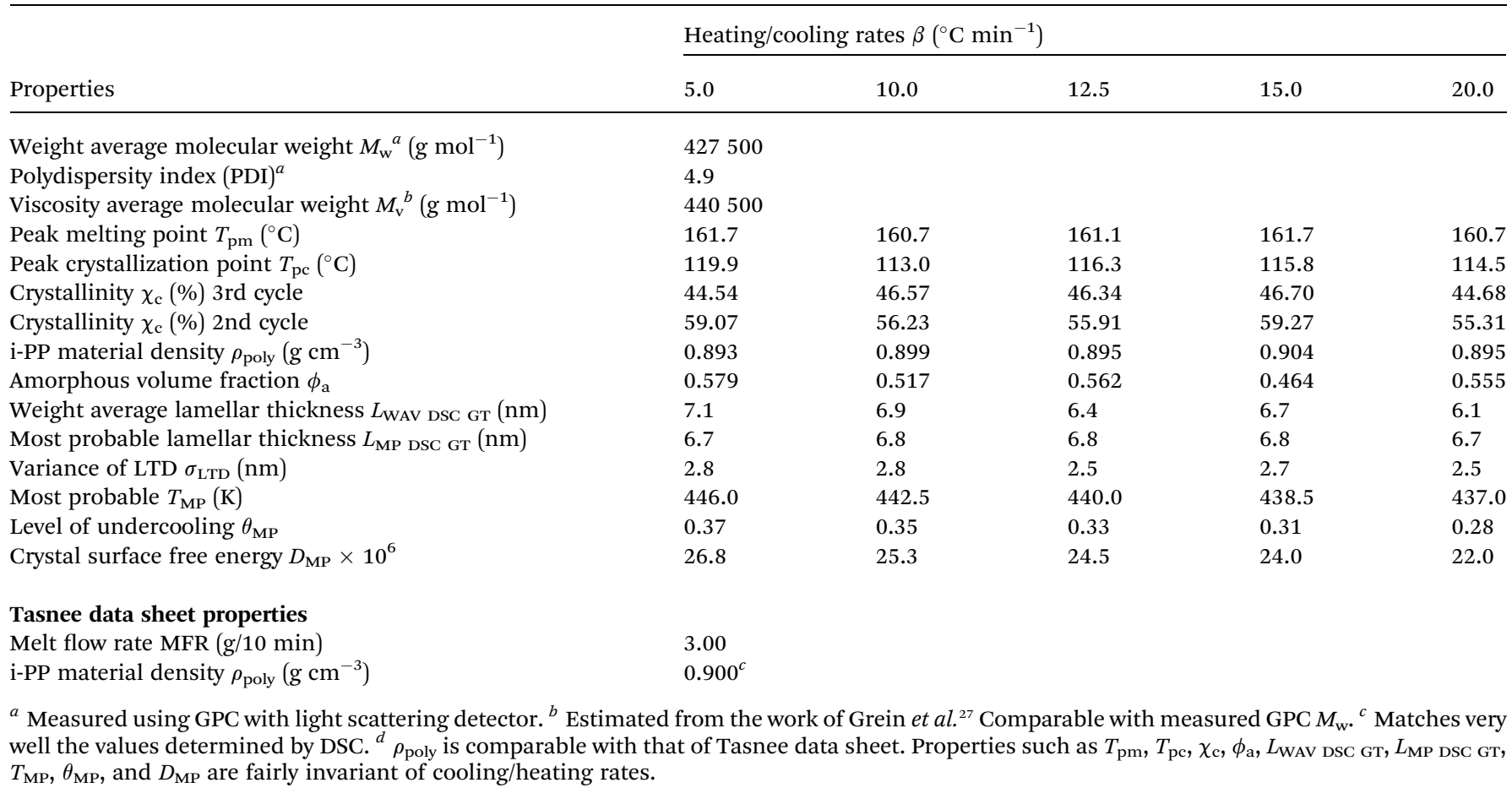

$0.850 \mathrm{~g} \mathrm{~mL} \mathrm{~m}^{-1} .^{30} \mathrm{Next}$, the amorphous volume fraction $\phi_{\mathrm{a}}$ was estimated using the relation $\phi_{\mathrm{a}}=\left(\rho_{\mathrm{c}}-\rho\right) /\left(\rho_{\mathrm{c}}-\rho_{\mathrm{a}}\right)$. Table 1 reports the above thermal properties of the experimental i-PP.

\subsection{Microstructure of the experimental i-PP}

The microstructural parameters (meso-pentad mole fractions) of the experimental i-PP were determined using ${ }^{13} \mathrm{C}$ NMR spectroscopy. For this, a Bruker $600 \mathrm{MHz}$ spectrometer was used. The sample was prepared at $120{ }^{\circ} \mathrm{C}$ by dissolving about $250 \mathrm{mg}$ of the sample in a $10 \mathrm{~mm}$ NMR tube using $3 \mathrm{~mL}$ of the deuterated dimethyl sulfoxide $\left(\mathrm{DMSO}-\mathrm{d}_{6}\right) /$ trichlorobenzene
(TCB) $10 / 90 \%(\mathrm{v} / \mathrm{v})$ mixed solvent. The DMSO- $\mathrm{d}_{6}$ was used as an internal lock.

The spectrum was recorded at $120{ }^{\circ} \mathrm{C}$ using WALTZ-16 decoupler, $30^{\circ}$ pulse angle with pulse repetition time of $10 \mathrm{~s}$. The free induction decays (FIDs) were stored in $32 \mathrm{~K}$ data points using a spectral width of $70 \mathrm{ppm}$. The experimental data were processed and analyzed using TOPSPIN software (version 2.0).

The spectral region of methyl carbon signals (22.60-19.50 ppm) was divided into three triad configurational sequences, that is, mm (22.60-21.10 ppm), mr (21.10-21.40 ppm), and rr (21.40-19.50 ppm). Each of the above triad regions was further split into three pentad configurational sequences using

Table 2 Mole fraction of configurational meso-pentad sequences in the experimental i-PP ${ }^{a}$

\begin{tabular}{|c|c|c|c|}
\hline \multicolumn{2}{|c|}{ Configurational meso-pentad sequences } & \multirow{2}{*}{$\begin{array}{l}\text { Chemical shift (in ppm) } \\
21.75\end{array}$} & \multirow{2}{*}{$\frac{\text { Mole fractions }}{0.855^{*}}$} \\
\hline $\mathrm{mmmm}$ & mm-centered & & \\
\hline $\mathrm{mmmr}$ & mm-centered & 21.56 & 0.028 \\
\hline rmmr & mm-centered & 21.31 & 0.004 \\
\hline mmrr & mr-centered & 20.02 & 0.032 \\
\hline $\mathrm{mmrm}+\mathrm{rmrr}$ & mr-centered & 20.87 & 0.016 \\
\hline rmrm & mr-centered & 20.72 & 0.013 \\
\hline rrrr & rr-centered & 20.35 & 0.011 \\
\hline mrrr & rr-centered & 20.22 & 0.014 \\
\hline mrrm & rr-centered & 19.92 & 0.027 \\
\hline
\end{tabular}

${ }^{a}$ MSL (meso-pentad sequence length) $=\frac{\mathrm{mmmm}+\frac{3}{2} \mathrm{mrrr}+2 \mathrm{rmmr}+\frac{1}{2} \mathrm{rmrm}+\frac{1}{2} \mathrm{rmrr}}{\frac{1}{2} \mathrm{mmmr}+\mathrm{rmmr}+\frac{1}{2} \mathrm{rmrm}+\frac{1}{2} \mathrm{rmrr}}=31$. The signals of mmrm and rmrr pentads overlap in the spectrum; hence, the sum ( $\mathrm{mmrm}+\mathrm{rmrr}$ ) is obtained. To calculate MSL, the contribution of rmrr has been taken equal to that of rmrr. ${ }^{34}$ MSL is also called average isotactic sequence length. ${ }^{*} X_{\mathrm{mmmm}}=0.855$. 
Lorentzian distribution functions. ${ }^{33}$ All the pentad sequence assignments and their mole fractions are listed in Table 2 . These were used to calculate the meso-pentad sequence length $(\mathrm{MSL})^{34}$ which is also called average isotactic sequence length.

\section{Theoretical}

\subsection{Modeling of polypropylene crystallization kinetics}

A new nonisothermal crystallization model $^{15}$ for crystalline polymer using the Avrami-Erofeev equation was published by us in 2013. This model, with detailed assumptions and mathematical derivation, is reported in the above reference. Here, a summary is provided as follows.

The nonisothermal Avrami-Erofeev polymer crystallization rate, involving nucleation and growth, can be written as:

$$
\frac{\mathrm{d} \alpha}{\mathrm{d} T}=\frac{k_{0}}{\beta} \times \exp \left[-\frac{E_{\mathrm{a}}}{R}\left(\frac{1}{T}-\frac{1}{T_{0}}\right)\right] \times n(1-\alpha)[-\ln (1-\alpha)]^{\frac{n-1}{n}}
$$

where we define the following:

$$
\begin{gathered}
f(\alpha)=n(1-\alpha)[-\ln (1-\alpha)]^{\frac{n-1}{n}} \\
k^{\prime}(T)=k_{0} \times \exp \left[-\frac{E_{\mathrm{a}}}{R}\left(\frac{1}{T}-\frac{1}{T_{0}}\right)\right]
\end{gathered}
$$

$$
E_{\text {a }} \text { (apparent crystallization energy) }=E_{\text {grow }}-E_{\text {nucl }}
$$

$k_{0}($ overall crystallization frequency factor $)=$

$$
\begin{array}{r}
\left(\frac{k_{\mathrm{s}} N_{0}}{V_{0}}\right) \frac{k_{\text {grow }, 0}}{k_{\text {nucl }, 0}} \neq f(T) \\
k_{\text {grow }}(T)=k_{\text {grow }, 0} \times \exp \left[-\frac{E_{\text {grow }}}{R}\left(\frac{1}{T}-\frac{1}{T_{0}}\right)\right] \\
k_{\text {nucl }}(T)=k_{\text {nucl }, 0} \times \exp \left[-\frac{E_{\text {nucl }}}{R}\left(\frac{1}{T}-\frac{1}{T_{0}}\right)\right]
\end{array}
$$

In the above equations, $f(\alpha)$ is called Avrami-Erofeev nonisothermal crystallization function, and $\alpha$ is the temperature- or time-dependent volume fraction of the molten polymer solidified due to cooling. Therefore, $\alpha$ concerns the phase morphology of the whole sample (melt plus solid). It is called relative crystallinity or degree of crystallization. $\beta$ is the cooling rate. $n$ is the dimension of the growing crystal. $N_{\mathrm{o}}$ is the number of germ nuclei, that is, the potential nucleus formation sites/ defects. $V_{\mathrm{o}}$ is the initial volume of the molten polymer. $K_{\mathrm{s}}$ is the shape factor for the growing nuclei. $k_{\text {grow, } 0}$ and $E_{\text {grow }}$ are the frequency factors and activation energy of crystal growth, respectively. $K_{\text {nucl,o }}$ and $E_{\text {nucl }}$ represent the corresponding terms for nucleation, respectively. $R$ is the universal gas constant, and $T_{\mathrm{o}}$ is the centering temperature.

The Avrami index $n$, in eqn (1), illustrates two aspects-the crystal dimension and the nature of nucleation process. Therefore, $n$ is written in terms of the following two components: ${ }^{35}$

$$
n=n_{\mathrm{d}}+n_{\mathrm{n}}
$$

where $n_{\mathrm{d}}$ shows the dimension of the growing crystals. Theoretically speaking, $n_{\mathrm{d}}$ can be only integers-1, 2, and 3-that correspond to one-, two-, and three-dimensional crystals formed, respectively; and $n_{\mathrm{n}}$ represents the nucleation process. In principle, it should be 0 or 1 , where 0 refers to instantaneous (athermal/heterogeneous) nucleation; and 1, to sporadic (thermal/homogeneous) one. For many systems, the modelpredicted $n$ turns out to be a non-integer which is attributed to $0 \leq n_{\mathrm{n}} \leq 1$. This means the simultaneous occurrence of instantaneous and sporadic nucleation processes. ${ }^{36} \mathrm{~A}$ balance between thermodynamic and kinetic factors influences the value of $n_{\mathrm{n}} \cdot{ }^{37}$

$\alpha(T)$ is related to the corresponding weight fraction relative crystallinity $\alpha_{\mathrm{w}}(T)$ through the following expression: ${ }^{15}$

$$
\alpha=\frac{\alpha_{\mathrm{w}}}{\alpha_{\mathrm{w}}+\rho_{\mathrm{c}} / \rho_{\mathrm{a}}\left(1-\alpha_{\mathrm{w}}\right)}
$$

where $\rho_{\mathrm{c}}$ and $\rho_{\mathrm{a}}$ are the densities of the crystalline and amorphous phases, respectively. For polypropylene, the values of $\rho_{\mathrm{c}}$ and $\rho_{\mathrm{a}}$ have been reported earlier. The relation $\alpha$ versus $T$ is called relative crystallinity or degree of crystallization profile.

$\alpha_{\mathrm{w}}$ can be calculated from a typical constant cooling rate nonisothermal DSC experiment by using eqn (10):

$$
\alpha_{\mathrm{w}}=\frac{\Delta H(T)}{\Delta H_{\text {total }}}=\frac{\int_{T_{i}}^{T}\left(\frac{\mathrm{d} H}{\mathrm{~d} T}\right) \mathrm{d} T}{\int_{T_{i}}^{T_{\infty}}\left(\frac{\mathrm{d} H}{\mathrm{~d} T}\right) \mathrm{d} T}
$$

where $\Delta H_{\text {total }}$ is the maximum enthalpy value reached at the end of the nonisothermal crystallization process and $\Delta H(T)$ is the enthalpy evolved as a function of crystallization temperature $T$. $T_{i}$ and $T_{\infty}$ represent the crystallization start and completion temperatures, respectively. $\Delta H_{\text {total }}$ and $\Delta H(T)$ both can be acquired through the software of a standard differential scanning calorimeter (DSC). Using eqn (9), the DSC-generated $\alpha_{\mathrm{w}}$ can be converted to the corresponding volume fraction $\alpha$.

The experimental confirmation of the above new nonisothermal crystallization model is available in one of our recent publications. ${ }^{25}$ See Harkin-Jones et al. ${ }^{3}$ that comprehensively reviews the literature models.

\subsection{Polypropylene melt behavior and crystallization: Flory's equilibrium theory treatment}

Here, we first summarize the microstructural defects of a typical Ziegler-Natta (Z-N) i-PP. Ti-based Z-N catalysts, in the presence of internal and external donors, synthesize i-PP predominantly with stereo-defects introduced in the crystallizable isotactic propylene sequence of the backbone. ${ }^{38-40}$ The origin of stereodefects has been elaborated elsewhere in the literature. ${ }^{41}$ This makes the i-PP backbone resembling that of, for example, a typical ethylene- $\alpha$-olefin copolymer where the $\alpha$-olefin introduces branching defects in the ethylene sequence of the backbone. Hence, an i-PP can be microstructurally defined to be a random stereo-copolymer with configurational defects along 
the backbone. ${ }^{\mathbf{4 0 - 4 2}}$ See Scheme 1. Accordingly, the i-PP stereodefect disrupts the backbone as the addition of the $\alpha$-olefin does in polyethylene. ${ }^{41}$ Therefore, Flory's copolymer equilibrium theory ${ }^{16-18}$ can also be applied to the stereo-irregular Z-N i-PP. ${ }^{40}$

In a random ethylene- $\alpha$-olefin copolymer, the ethylene perpetuation probability $p$, that is, the probability that a crystallizable unit is succeeded by another such unit is given by $\left[X_{\mathrm{E}}+\right.$ $\left.\left(1-X_{\mathrm{E}}\right) / 2\right]$ where $X_{\mathrm{E}}$ is the average ethylene mole fraction. ${ }^{\mathbf{1 9 , 4 3}}$ The term $\left(1-X_{\mathrm{E}}\right) / 2$ is the correction due to the incorporation of one $-\mathrm{CH}_{2}$ - unit (or half $\mathrm{C}_{2} \mathrm{H}_{4}$ unit) per insertion of one $\alpha$-olefin comonomer and exclusion of the pendant alkyl group (greater than methyl) from the chain fold. As per Scheme 1 and what is stated above, for an i-PP, the analogous propylene perpetuation probability $p$ can be approximated by the crystallizable isotacticity index $X_{\text {mmmm }}$ (meso-pentad) mole fraction.

According to the above description and Flory's copolymer equilibrium theory, the propylene perpetuation probability $p$ can be related to the critical (limiting) sequence number $n^{*}(T)$ of the stable crystallite, that equilibrates with the melt at a given temperature, using the following equations: ${ }^{16-18}$

$$
\begin{aligned}
n^{*}(T)= & \frac{-1}{\theta+\ln p}\left[\ln \left(\frac{D X_{\mathrm{mmmm}}}{p}\right)+2 \ln \left(\frac{1-p}{1-\mathrm{e}^{-\theta}}\right)\right] \\
& \times(\text { dimensionless })
\end{aligned}
$$

$$
\begin{aligned}
\theta \text { (level of undercooling }) & =\frac{\Delta H_{\mathrm{u}}}{R T_{\mathrm{m}}^{0}} \frac{T_{\mathrm{m}}^{0}-T}{T} \\
& =\frac{\Delta H_{\mathrm{u}}}{R T_{\mathrm{m}}^{0}} \frac{\Delta T}{T} \text { (dimensionless) }
\end{aligned}
$$

$D$ (crystal surface free energy effect)

$$
=\mathrm{e}^{-2 \sigma_{\mathrm{e}} a_{\mathrm{o}} / R T} \text { (dimensionless) }
$$

where $X_{\text {mmmm }}$ is the mole fraction of the crystallizable (mesopentad) isotacticity index; $R$ is the universal gas constant; and $T$ is cooling temperature in $\mathrm{K}$.

Note that $n^{*}, \theta$, and $D$ are dimensionless. Therefore, they offer a common footing to compare the melt behavior and crystallization of i-PPs of different structures under varying
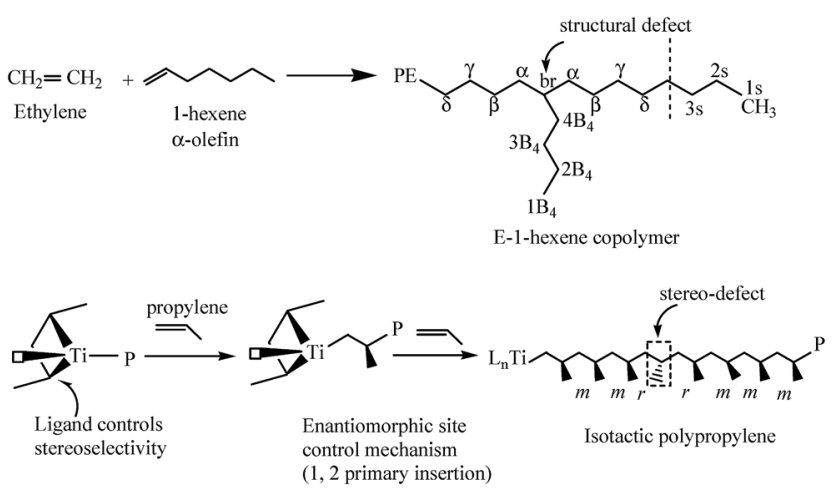

Scheme 1 Comparison of the stereo-defect of an i-PP with the structural defect of a typical ethylene- $\alpha$-olefin copolymer. The i-PP stereo-defects originate from enantiofacial error, chain-end epimerization, and chain-end effect. ${ }^{41}$ experimental conditions. In eqn $(12), T_{\mathrm{m}}^{0}(459.1 \mathrm{~K})$ is the melting point of an isotactic polypropylene perfect crystal (completely defect-free i-PP of infinite lamellar thickness and molar mass). This is also called polypropylene equilibrium melting point. $\Delta H_{\mathrm{u}}\left(2100 \mathrm{cal} \mathrm{mol}^{-1}=2 \times 10^{9} \mathrm{erg} \mathrm{cm}^{-3}\right)$ is the heat of fusion for perfect crystal i-PP repeat unit $-\left[\mathrm{CH}_{2}-\mathrm{CH}\left(\mathrm{CH}_{3}\right)\right]-, \sigma_{\mathrm{e}}$ (96 erg $\mathrm{cm}^{-3}$ ) is the polypropylene basal/fold free surface energy, and $a_{\mathrm{o}}\left(2.05 \times 10^{5} \mathrm{~m}^{2} \mathrm{~mol}^{-1}\right)$ is the cross-sectional area of a polypropylene chain. ${ }^{16,30,31}$ All these properties relate to alpha-phase i-PP. For a constant cooling rate nonisothermal DSC crystallization process, $n^{*}(T)$ evaluates the temperaturedependent dynamic critical crystallite stability. Only isotactic propylene sequences, greater than $n^{*}(T)$, form stable crystals. With increasing temperature, the crystals melt and $n^{*}(T)$ increases, and $n^{*} \rightarrow \infty$ represents the thickest possible crystals. ${ }^{18}$

\section{Results and discussion}

\subsection{Crystallization kinetics of the experimental isotactic polypropylene}

In this section, we address the nonisothermal crystallization kinetics of the experimental i-PP in terms of apparent crystallization activation energy $E_{\mathrm{a}}$, frequency factor $k_{0}$, crystal dimension $n_{\mathrm{d}}$, and nucleation mode $n_{\mathrm{c}}$. We discuss them considering five different Cycle 2 DSC cooling rates.

4.1.1 Solution of crystallization model equation and estimation of kinetic triplet. We solved eqn (1) numerically, and estimated the kinetic triplet $\left(k_{0}, n\right.$, and $\left.E_{\mathrm{a}}\right)$ as follows. First, we modified it through separation of variables, and integrated the left hand side (LHS) analytically, and transformed the right hand side (RHS) into the well-known temperature integral $\int \exp \left(-\frac{E_{\mathrm{a}}}{R T}\right) \mathrm{d} T$. See eqn (14) and (15). Next, we converted the temperature integral into a real part and an exponential integral part. ${ }^{44}$ See eqn (16). This is how we integrated the temperature integral, which we finally transformed to the non-linear algebraic form. We solved this modified model equation using the Mathematica 8.0 Nonlinear Model Fit software. $T_{\mathrm{o}}=370 \mathrm{~K}$ was used as the centering temperature. The LHS of eqn (16), containing $-\ln (1-\alpha)$, has a point of discontinuity at $\alpha_{\text {final }}=1$. This was resolved by approximating $\alpha_{\text {final }} \cong 0.9999$. Depending on the cooling rates, 30 to 45 experimental data points were considered for kinetic parameter estimation.

$$
\int \frac{\mathrm{d} \alpha}{n(1-\alpha)[-\ln (1-\alpha)]^{\frac{n-1}{n}}}=\int \frac{k_{0}}{\beta} \times \exp \left[-\frac{E_{\mathrm{a}}}{R}\left(\frac{1}{T}-\frac{1}{T_{0}}\right)\right] \mathrm{d} T
$$

$$
\begin{gathered}
{[-\ln (1-\alpha)]^{\frac{1}{n}}=\frac{k_{0} \exp \left(\frac{E_{\mathrm{a}}}{R T_{0}}\right)}{\beta} \int \exp \left(-\frac{E_{\mathrm{a}}}{R T}\right) \mathrm{d} T} \\
{[-\ln (1-\alpha)]^{\frac{1}{n}}=\frac{k_{0} \exp \left(\frac{E_{\mathrm{a}}}{R T_{0}}\right)}{\beta}\left[T \exp \left(-\frac{E_{\mathrm{a}}}{R T}\right)+\frac{E_{\mathrm{a}} E_{\mathrm{i}}\left(-\frac{E_{\mathrm{a}}}{R T}\right)}{R}\right]}
\end{gathered}
$$


Table 3 Model-predicted crystallization kinetic parameters

\begin{tabular}{llc}
\hline $\begin{array}{l}\text { DSC cooling } \\
\text { rates }{ }^{\circ} \mathrm{C} \mathrm{min}\end{array}$ & $\begin{array}{l}\text { Crystallization } \\
\text { kinetics parameters }\end{array}$ & i-PP homopolymer \\
\hline 5.0 & $n$ & 3.50 \\
& $k_{0}\left(\mathrm{~min}^{-1}\right)$ & 0.20 \\
& $E_{\mathrm{a}}\left(\mathrm{kJ} \mathrm{mol}^{-1}\right)$ & 123.06 \\
& $R^{2}$ & 0.9904 \\
10.0 & $n$ & 3.00 \\
& $k_{0}\left(\mathrm{~min}^{-1}\right)$ & 0.54 \\
& $E_{\mathrm{a}}\left(\mathrm{kJ} \mathrm{mol}^{-1}\right)$ & 123.63 \\
& $R^{2}$ & 0.9891 \\
12.5 & $n$ & 2.60 \\
& $k_{0}\left(\mathrm{~min}^{-1}\right)$ & 0.75 \\
& $E_{\mathrm{a}}\left(\mathrm{kJ} \mathrm{mol}^{-1}\right)$ & 123.55 \\
& $R^{2}$ & 0.9858 \\
15.0 & $n$ & 2.38 \\
& $k_{0}\left(\mathrm{~min}^{-1}\right)$ & 1.02 \\
& $E_{\mathrm{a}}\left(\mathrm{kJ} \mathrm{mol}^{-1}\right)$ & 123.71 \\
& $R^{2}$ & 0.9886 \\
& $n$ & 2.13 \\
& $n$ & 1.69 \\
& $k_{0}\left(\mathrm{~min}^{-1}\right)$ & 123.56 \\
& $E_{\mathrm{a}}\left(\mathrm{kJ} \mathrm{mol}^{-1}\right)$ & 0.9881 \\
& $R^{2}$ &
\end{tabular}

where $E_{\mathrm{i}}\left(-\frac{E_{\mathrm{a}}}{R T}\right)$ is the exponential integral of $-\frac{E_{\mathrm{a}}}{R T}$.

We estimated the model parameters considering the following statistical criteria-95\% confidence interval, coefficient of determination $\left(R^{2}\right)$, estimated variance, and standard error. The aforementioned Mathematica software eventually generates them. Convergence was accepted when the objective function changed less than the specified tolerance of $10^{-8}$. For the sake of brevity and sufficiency, we list only $R^{2}$ for each of the estimated kinetic parameters. See Table 3.

4.1.2 Evaluation of model performance and significance of the predicted results. Now we evaluate the present nonisothermal Avrami-Erofeev model performance, and discuss the significance of the major pertinent results. Fig. 1 compares the model-predicted relative crystallization profiles, determined using eqn (1), with the corresponding DSC profiles at different cooling rates. The agreement between model and experiment, in each case, is excellent. The crystallization profiles shifted leftward with the increase in cooling rate $\beta$. This trend matches that of the cooling rate-dependent nonisothermal i-PP relative crystallinity profiles reported in the literature., ${ }^{3,5}$ Our premier model finding is the following. A single value of $n$ represents the whole crystallization regime (primary as well as secondary). This result signifies that the same mechanism of nucleation and crystal growth holds, irrespective of the cooling rates, all throughout the nonisothermal i-PP crystallization. This argues how the present model overcomes the drawbacks and limitations of the arbitrary parameter-based existing nonisothermal crystallization models, summarized in our previous study. ${ }^{15}$ Also, see Harkin-Jones et al. $^{3}$ that reviews the parametric nonisothermal crystallization literature models. Therefore, the assumption of change in crystallization mechanism, as reported in the literature, ${ }^{3,45-48}$ is invalid.

Table 3 shows that the model-predicted $n_{\mathrm{d}}$ ranges between 2 and 3 , and it depends on the cooling rates. For $\beta=5.0$ and $10.0^{\circ} \mathrm{C} \mathrm{min}^{-1}, n_{\mathrm{d}}=3$; and for $\beta=12.5,15.0$, and $20.0^{\circ} \mathrm{C} \mathrm{min}^{-1}$, $n_{\mathrm{d}}=2$. Hence, the resulting i-PP crystal dimension varies between spherical and cylindrical, that is, two and three dimensional alignments of the polymer backbone lamellae, corresponding to low and high cooling rates, respectively. Further, $n$ has an integral part $n_{\mathrm{d}}$ and a fractional part $n_{\mathrm{n}}$. Therefore, according to eqn (8), for the subject i-PP, instantaneous (athermal/heterogeneous) as well as sporadic (thermal/ homogeneous) nucleations ( $\left.0.00 \leq n_{\mathrm{n}} \leq 0.60\right)$ simultaneously prevail under the experimental cooling rates. The conformation of the $-\mathrm{CH}_{3}$ group, that is, the i-PP backbone stereo-defect does not deflect this model-prediction. Consider particularly the $5.0{ }^{\circ} \mathrm{C} \min ^{-1}$ model prediction in Table 3. Here, $n_{\mathrm{d}}=3$ which

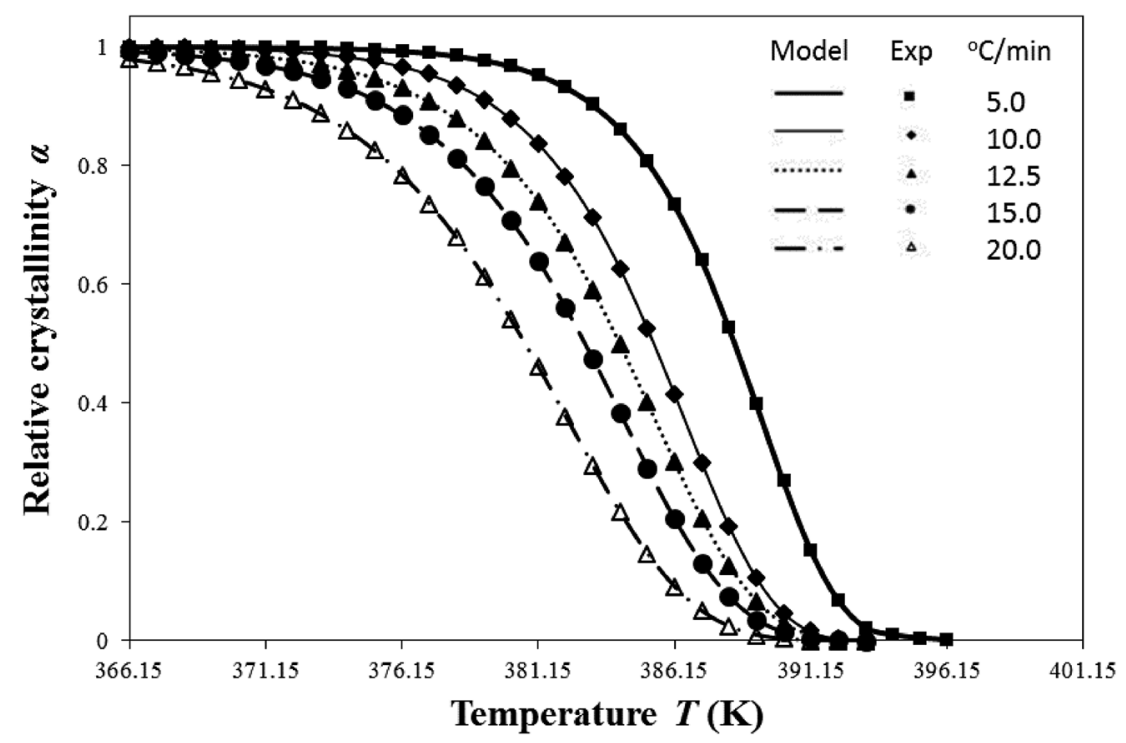

Fig. 1 Comparison of model-predicted i-PP relative crystallinity profiles with DSC experiments for different cooling rates. 


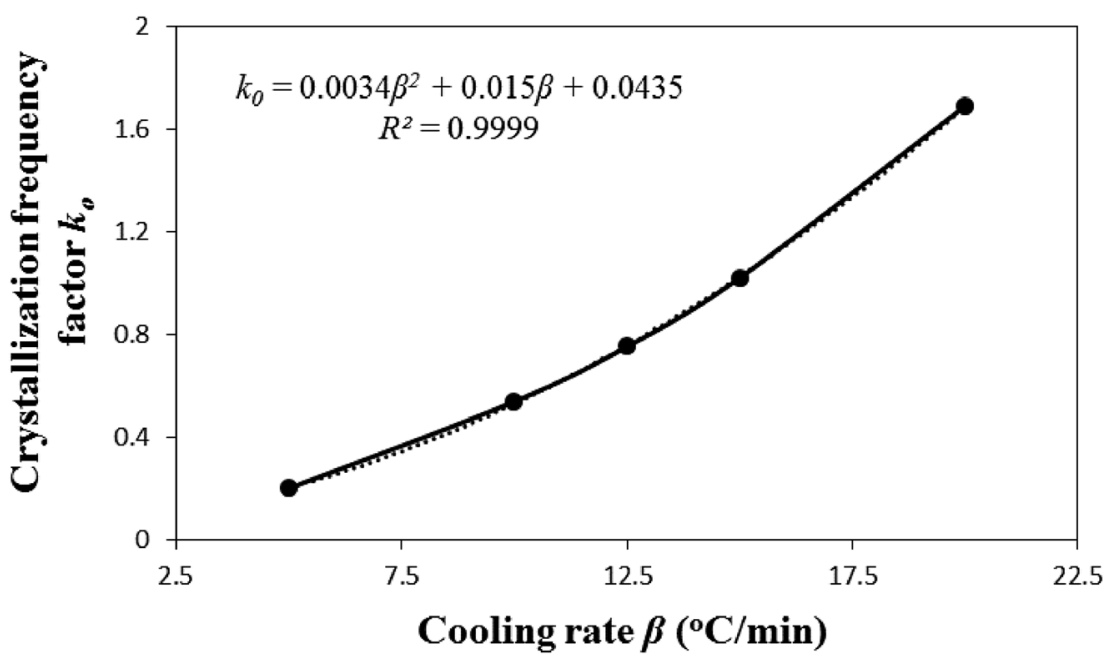

Fig. 2 Effect of cooling rates on i-PP nonisothermal crystallization frequency factor $k_{0}$.

indicates the formation of spherulite i-PP crystals. This model finding matches what Sajkiewicz et $a l .{ }^{4}$ experimentally observed for crystallization of pristine i-PP at the above cooling rate, using polarized light microscopy.

The crystallization profiles- $\alpha$ versus $T$ relations-feature very fast primary and slow (impinged) secondary crystallizations. We shall discuss them from the perspective of Flory's equlibrium theory, particularly in terms of the level of dimensionless undercooling $\theta$ and the crystal surface free energy $D$, at a later section.

Fig. 1 also confirms that a single $E_{\mathrm{a}}$ fits the well-known isokinetic Avrami-Erofeev crystallization mechanism throughout the entire i-PP crystallization process (primary plus secondary crystallization). Therefore, $E_{\mathrm{a}}$ is essentially constant of crystallization time or temperature and relative crystallinity $\alpha$. Now we compare this finding with that published in the literature.

First, we consider the report by Harkin-Jones et al. ${ }^{3}$ who modeled nonisothermal DSC crystallization of an un-nucleated i-PP (FINA 4060S, MFR $=3.00 \mathrm{~g} / 10 \mathrm{~min}$ at $230.0{ }^{\circ} \mathrm{C}$ and $2.16 \mathrm{~kg}$ load). Note that this MFR equals to that of our experimental sample. They evaluated a number of literature models, all of which incorporate arbitrary curve-fitting parameters. The twoparameter modified Ozawa model (with induction period), extended by Hammami, Spruiell, and Mehrotra ${ }^{49}$ performed the best.

Second, Zheng et al. ${ }^{5}$ modeled the nonisothermal crystallization of i-PP (MFR $=3.88 \mathrm{~g} / 10 \mathrm{~min}$ at $230.0{ }^{\circ} \mathrm{C}$ and $2.16 \mathrm{~kg}$ load) considering an empirical nuclei density function, induction period, and temperature-dependent Hoffman-Lauritzen spherulite growth rate. This heuristic simulation model has the following limitations. It does not represent secondary crystallization for cooling rates exceeding $5.0{ }^{\circ} \mathrm{C} \mathrm{min}{ }^{-1}$. It also shows that effective activation energy varies with relative crystallinity $\alpha$.

Both the above curve-fitting models have unfortunately no mechanistic, kinetic, and thermodynamic basis. Hence, the model parameters and predictions, unlike those of ours, are of limited physical significance.
Third, Supaphol et al. ${ }^{50}$ modeled the nonisothermal crystallization activation energy of selected aromatic polyesters and showed that it is a function of the relative crystallinity $\alpha$. Depending on the polymer structure, it either monotonically increases, or it first increases and then it decreases as $\alpha$ increases. This variational trend was ascribed to the dependence of nucleation energy barrier on temperature. However, note that this explanation ignores isokinetic concept, crystal growth, as well as primary and secondary crystallizations. Hence, their model prediction and explanation are insufficient and unacceptable.

Fig. 2 illustrates that the model-predicted nonisothermal crystallization frequency factor $k_{0}$ [defined by eqn (5)], for the experimental i-PP, well correlates to the cooling rate $\beta\left(R^{2}=\right.$ $0.9999) . k_{0}$ progressively increases as $\beta$ increases. Our physical interpretation of this correlation follows. Considering the relation between kinetics and thermodynamics, it can be shown that $k_{0} \Rightarrow \exp (S / R)$ or $S \Rightarrow R \ln k_{0}$ where $S$ is entropy of the system and $R$ is the universal gas constant. ${ }^{51}$ Therefore, the entropy of the experimental i-PP nonisothermal crystallization increases as $k_{0}$ and the cooling rate $\beta$ increase. $k_{0}$ is, therefore,

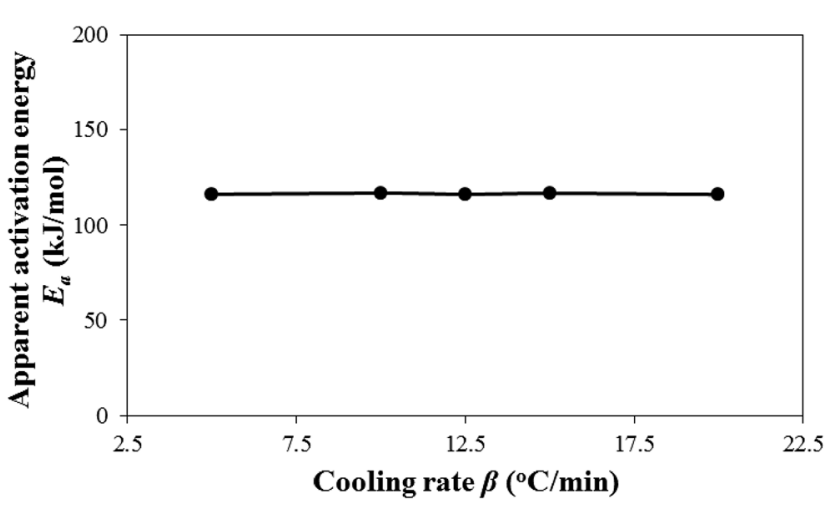

Fig. 3 Effect of cooling rates on i-PP nonisothermal crystallization apparent activation energy. 
a measure of entropy (system disorder) and it is cooling ratedependent. Now, we revisit the growth of crystal dimension $n_{\mathrm{d}}$ from the thermodynamic entropy perspective. Recall the results reported in Table 3. Low and increasing system disorders, among other factors, favor the growth of spherulitic and cylindrical crystals, respectively. This is a new model prediction for nonisothermal i-PP crystallization.

Fig. 3 shows the effect of cooling rates on the modelpredicted apparent activation energy $E_{\mathrm{a}}=E_{\text {grow }}-E_{\text {nucl }}$. This is a major motif of this study. $E_{\mathrm{a}}$ does not practically vary with cooling rate $\beta$. Hence, it, unlike $k_{0}$, is entropy-independent for the experimental i-PP nonisothermal crystallization. This result particularly differs from what Sahay and Krishnan have reported regarding the influence of $\beta$ on activation energy of aromatic polyester crystallization. ${ }^{52}$ To fit the DSC data, these authors considered an effective activation energy $E_{\text {ea }}$ that consists of cooling rate-independent activation energy $E_{0}$ and cooling rate-dependent activation energy $E_{1}$, which are related to each other as follows: $E_{\text {ea }}=E_{0}+E_{1} \ln (A \beta)$, where $A$ is an arbitrary constant. Regrettably, this treatment, like the model by Hammami, Spruiell, and Mehrotra. ${ }^{49}$ has no mechanistic, kinetic, and thermodynamic basis. This is a purely data-fitting approach. Hence, the above effective crystallization activation energy concept cannot be accepted. By contrast, our model duly considers isokinetic concept, crystal growth, as well as primary and secondary i-PP crystallizations; and mathematically shows that the activation energy is temperature-, entropy-, and cooling rate-independent. The pendant $-\mathrm{CH}_{3}$ (methyl) group of i-PP, with that of the stereo-defect, despite being inclusive in the crystal lamella, ${ }^{\mathbf{1 8 3 8 , 4 2}}$ does not affect the invariance of $E_{\mathrm{a}}$. Fig. 4 shows the qualitative stereo-defect distribution (SDD) of the experimental i-PP. This was determined using Crystaf and following the procedure reported in the literature. ${ }^{53}$ The SDD is segregated mainly into the following two regions: (i) the defectrich atactic (amorphous) soluble content (4.0 wt\%), and (ii) defect-lean highly isotactic crystalline backbones (around peak crystallization temperature of $83.2^{\circ} \mathrm{C}$ in TCB). The interference of the former into the melt crystallization of the latter, as per the model prediction, does not affect $E_{\mathrm{a}}$ either. The overall discrete meso-pentad distributions listed in Table 2 complement Fig. 4. They were determined using ${ }^{13} \mathrm{C}$ NMR spectroscopy. ${ }^{34,54}$

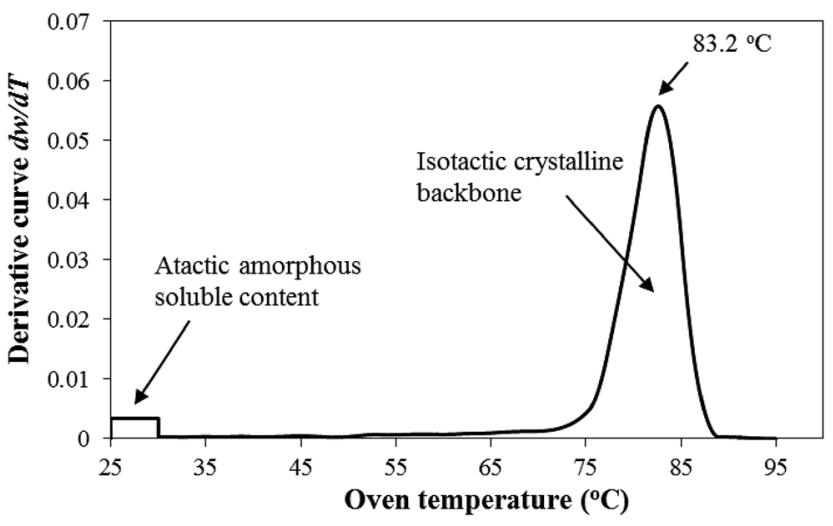

Fig. 4 Crystaf analysis of the experimental Ziegler-Natta i-PP.
Based on the overall findings of Fig. 1 and 3, this study confirms the invariance of activation energy articulated by Galwey and co-thinkers, ${ }^{55,56}$ and does not support the concept of variable instantaneous activation ${ }^{\mathbf{4 9 , 5 0 , 5 2 , 5 7 - 5 9}}$ which is practiced in analyzing nonisothermal crystallization kinetic data. This conclusion originates from the correct application of isokinetic concept and the current nonisothermal Avrami-Erofeev crystallization model, and the appropriate calculation algorithm that we developed. This is how we address in this study the mathematical artefact that exists in the literature.

Now, we evaluate the effect cooling rate $\beta$ on the rate of nucleation with respect to that of the crystal growth as follows.

Using eqn (4) to (7), we drive the following relation:

$\frac{1}{C} \frac{k_{\text {nucl }, 0}}{k_{\text {grow }, 0}}=\left[\frac{1}{k_{0}} \exp \left(\frac{E_{\mathrm{a}}}{R T_{0}}\right)\right] \times \exp \left(-\frac{E_{\mathrm{a}}}{R T}\right) \quad$ where $C=\frac{k_{\mathrm{s}} N_{0}}{V_{0}}$

We expand $\exp \left(-\frac{E_{\mathrm{a}}}{R T}\right)$ of eqn (17), according to Taylor
eries, as:

$$
\exp \left(-\frac{E_{\mathrm{a}}}{R T}\right)=1-\frac{E_{\mathrm{a}}}{R T}+\frac{1}{2 !}\left(\frac{E_{\mathrm{a}}}{R T}\right)^{2}-\frac{1}{3 !}\left(\frac{E_{\mathrm{a}}}{R T}\right)^{3}+\ldots
$$

We calculated $\frac{E_{\mathrm{a}}}{R T}$ (dimensionless apparent crystallization energy), as a function of $\beta$ for the experimental i-PP, using the present crystallization model-predicted $E_{\mathrm{a}}$ and the Cycle 2 DSC data. We found the following: $0.0355 \leq \frac{E_{\mathrm{a}}}{R T} \leq 0.0384$; hence, $\frac{E_{\mathrm{a}}}{R T} \ll 1$. So, eqn (18) can be written as $\exp \left(-\frac{E_{\mathrm{a}}}{R T}\right)=1-\frac{E_{\mathrm{a}}}{R T} \approx 1$. This reduces eqn (17) to $\frac{1}{C} \frac{k_{\text {nucl }, 0}}{k_{\text {grow }, 0}}=\frac{1}{k_{0}} \exp \left(\frac{E_{\mathrm{a}}}{R T_{0}}\right) \neq f(T, \theta$, and $D)$. Accordingly, we estimated $\frac{1}{C} \frac{k_{\text {nucl }, 0}}{k_{\text {grow }, 0}}$. Fig. 5 shows how $\beta$ affects $\frac{1}{C} \frac{k_{\text {nucl }, 0}}{k_{\text {grow }, 0}}$. The effect is more pronounced at lower value of $\beta$ such as $5.0{ }^{\circ} \mathrm{C} \min ^{-1}$; with the increase in $\beta, \frac{1}{C} \frac{k_{\text {nucl, }, 0}}{k_{\text {grow }, 0}}$ decreases and tends to assume an asymptotic value. $\frac{1}{C} \frac{k_{\text {nucl }, 0}}{k_{\text {grow }, 0}}$, as shown above, is inversely related to $k_{0}$ where $k_{0} \Rightarrow \exp (S / R)$ or $S \Rightarrow R \ln k_{0}$, and $k_{0}$ increases with $\beta$ (see Fig. 2). Therefore, low system entropic disorder, that corresponds to low $\beta$, increases the nucleation rate by several folds over the crystal growth rate. This effect, with the increase in $\beta$, that is, entropy shows to gradually diminish. The above finding is another manifest of the merit of the new crystallization model. Such a result does not appear in the literature.

Based on the preceding discussion, we conclude that the nonisothermal primary and secondary crystallizations of the experimental i-PP occur isokinetically with constant (temperature-, entropy-, and cooling rate-invariant) apparent kinetic energy. The crystal dimension varies between cylinder and sphere while instantaneous (athermal/heterogeneous) and 


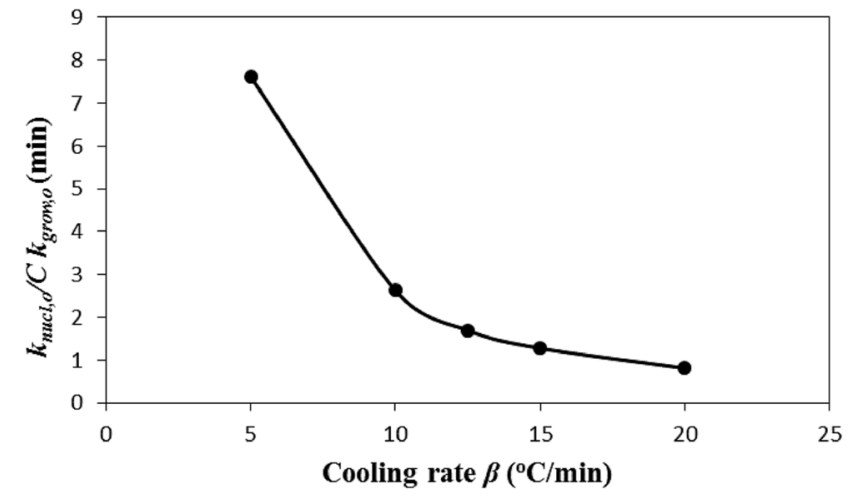

Fig. 5 Effect of cooling rate $\beta$ on $\frac{1}{C} \frac{k_{\text {nucl, }, 0}}{k_{\text {grow }, 0}}$ that predicts the rate of nucleation with respect to that of crystal growth.

sporadic (thermal/homogeneous) nucleations can co-occur. The cooling rate and system entropy influence the rate of nucleation over that of the crystal growth.

\subsection{Melting behavior and crystallization of i-PP: Flory's equilibrium theory perspective}

In this section, we discuss the melting behavior and crystallization of the experimental i-PP from Flory's equilibrium theory perspective, particularly considering the following three temperature-dependent dimensionless factors-critical stable crystallite sequence number $n^{*}$, level of undercooling $\theta$, and crystal surface free energy $D$. See eqn (11)-(13), respectively.

First, we evaluate the influence of the level of undercooling $\theta$ and crystal surface free energy $D$ on the nonisothermal crystallization of i-PP. $\theta$ and $D$ were calculated using eqn (12) and (13), respectively, and the Cycle 2 DSC data. Fig. 6 demonstrates that for a given cooling rate $\beta$ and beyond induction/nucleation regime $(\alpha=0.1)$, increasing $\theta$ sharply enhances the primary crystallization profile, and makes it proceed very fast. The plots shift rightward with the increase in $\beta$. Here, for a given value of the relative crystallinity (degree of crystallization) $\alpha, \theta$ increases as $\beta$ increases, without changing the apparent crystallization

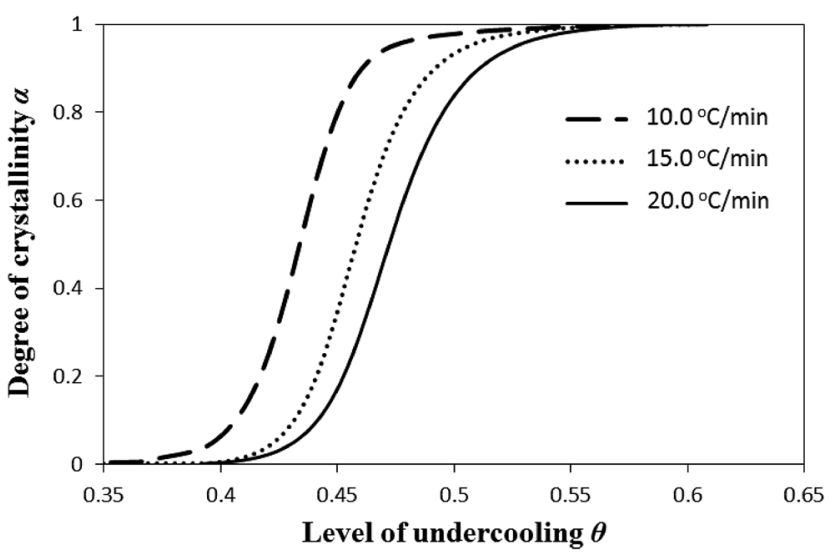

Fig. 6 Variation of relative crystallinity $\alpha$ with level of dimensionless undercooling $\theta$. energy $E_{\mathrm{a}}$. Also, see Fig. 2. On the other hand, the slower secondary crystallization (impingement of crystal growth) shows milder impact of $\theta$ on $\alpha$; $\alpha$ gradually increases with the increase in $\theta$. This finding differs from what we notice to happen in primary crystallization. The values of $\theta$ that correspond to the onset of primary and secondary crystallizations increase with the increase in $\beta$.

Fig. 7 investigates how crystal surface free energy $D$ affects the nonisothermal crystallization of i-PP. The overall impact is opposite to that of the level of undercooling $\theta$ on relative crystallinity $\alpha$. This is elaborated as follows. Here, a decrease in $D$ rapidly accelerates the primary crystallization $\alpha$. The plots shift leftward with the increase in the cooling rate $\beta$. For the same $\alpha$, $D$ decreases as $\beta$ increases, without affecting $E_{\mathrm{a}}$. On the contrary, during the slower secondary crystallization, $\alpha$ very gradually increases with the decrease in $D$. This trend deflects from the situation that prevails in primary crystallization. The onset secondary crystallization $D$ decreases with the increase in $\beta$.

From the above overall findings of Fig. 6 and 7, we conclude that primary and secondary crystallizations originate from the increase and decrease in the level of undercooling and crystal surface free energy, respectively. In primary crystallization, the $\mathrm{d} \alpha / \mathrm{d} \theta$ and $-\mathrm{d} \alpha / \mathrm{d} D$ are much higher than the corresponding derivatives in secondary crystallization. However, these two competitive temperature-dependent equilibrium theory parameters $(\theta$ and $D)$, despite having opposite variational trend, do not change the apparent crystallization energy $E_{\text {a }}$, either as a function of cooling temperature or rate. To the best of our knowledge, these are insightful new explanations for the observed characteristics of the fairly fast nonisothermal primary crystallization and very slow secondary crystallization of i-PP.

Fig. 8 is the consequence of the combined effect of the level of undercooling $\theta$ and crystal surface free energy $D$, expressed in terms of critical (limiting) sequence number $n^{*}$, on the nonisothermal crystallization of i-PP. See eqn (11); $X_{\mathrm{mmmm}}=0.855$ $\left({ }^{13} \mathrm{C}\right.$ NMR value); MSL (meso sequence length) $=31$ (Table 2). $X_{\mathrm{mmmm}}=0.855$ fairly matches the value that $T_{\mathrm{m}}=161.0^{\circ} \mathrm{C}$, the

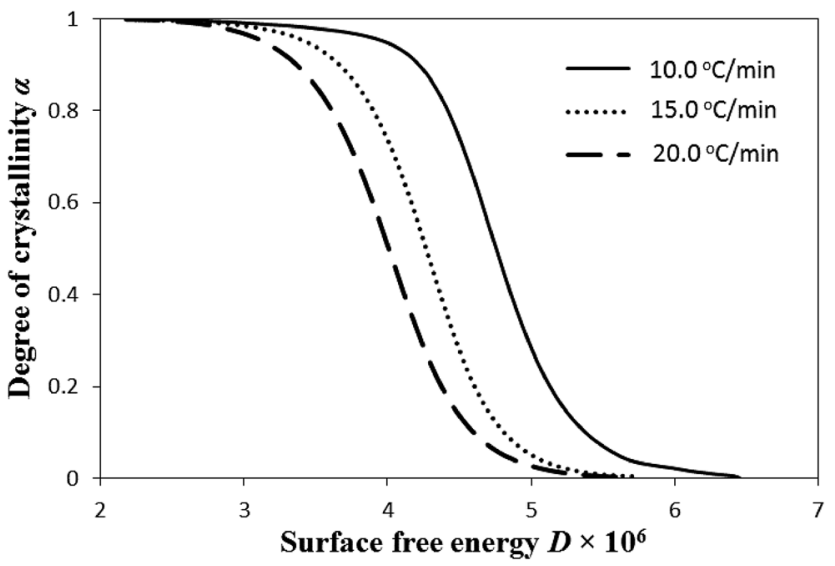

Fig. 7 Variation of relative crystallinity $\alpha$ with dimensionless crystal surface free energy $D$. 


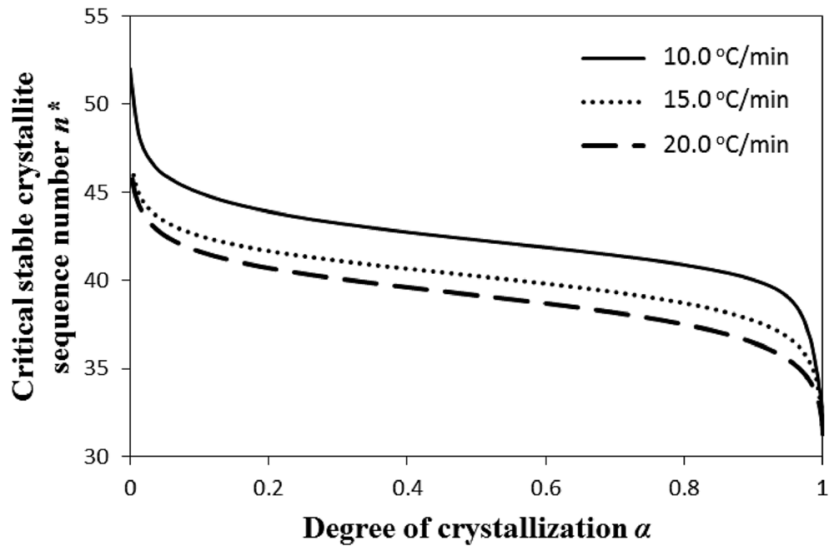

Fig. 8 Variation of critical crystallite sequence number $n *$ with relative crystallinity $\alpha$.

melting point of our experimental i-PP, reads from Fig. 4 of Zhang et al. ${ }^{60}$ Note that $n^{*}$ signifies the sequence of the i-PP repeat unit $-\left[\mathrm{CH}_{2}-\mathrm{CH}\left(\mathrm{CH}_{3}\right)\right]-$ of the stable crystallite, that equilibrates with the melt at a given melt temperature. Hence, it measures the temperature-dependent dynamic critical crystallite stability. This figure shows how $n^{*}$ varies as a function of $\alpha$ with the progressive development of the crystallinity profile. For a given cooling rate $\beta$ and beyond induction/nucleation regime $(\alpha=0.1), n^{*}$ fairly decreases as $\alpha$ sharply increases during primary crystallization. Here, for a given value of $\alpha, n^{*}$ increases with the decrease in cooling rate $\beta$. During secondary crystallization, $n^{*}$ steeply decreases as $\alpha$ slowly increases. All the plots shift rightward with the decrease in $\beta$. The $n^{*}$ versus $\alpha$ functional variation does not affect the apparent crystallization energy $E_{\mathrm{a}}$.

Fig. 9 compares the profiles of DSC-determined temperaturedependent instantaneous (dynamic) crystallinity $\chi(T)$ for different cooling rates. $\chi(T)$ was estimated using the following relation:

$$
\chi(T)=\frac{\Delta H(T)}{\Delta H_{\mathrm{f}}^{0}}=\frac{\int_{T_{\mathrm{i}}}^{T}\left(\frac{\mathrm{d} H}{\mathrm{~d} T}\right) \mathrm{d} T}{\Delta H_{\mathrm{f}}^{0}}
$$

where $\Delta H_{\mathrm{f}}^{\circ}$ is the heat of fusion $\left(8786.4 \mathrm{~J} \mathrm{~mol}^{-1}=207 \mathrm{~J} \mathrm{~g}^{-1}\right)$ of the perfect i-PP crystal (of infinite lamellar thickness and molar mass). Therefore, $\chi(T)$, unlike relative crystallinity $\alpha(T)$, is heat of fusion-based crystallinity that concerns the phases of the material solidified from the cooling melt.

The Cycle 2 DSC crystallization data were applied to calculate $\chi(T)$. For each cooling rate $\beta$, the $\chi$ versus $T$ relation shows the following common trend. $\chi$ initially increases fairly sharply as $T$ decreases with continued cooling. However, below a critical cooling temperature $T_{\text {c,critical }}$, it asymptotically flattens (which indicates hindrance (impingement) to the further development of crystallinity), and is not any further affected by decreasing $T$ and hence, the level of undercooling $\theta$ and crystal surface free energy $D$. At $T<T_{\mathrm{c} \text {,critical }}$, defect-rich i-PP chains, having slower melt chain dynamics, crystallize. ${ }^{16} T_{\mathrm{c}, \text { critical }}$ decreases with the increase in $\beta$. Only above $T_{\mathrm{c}, \text { critical }}$, the following happens:

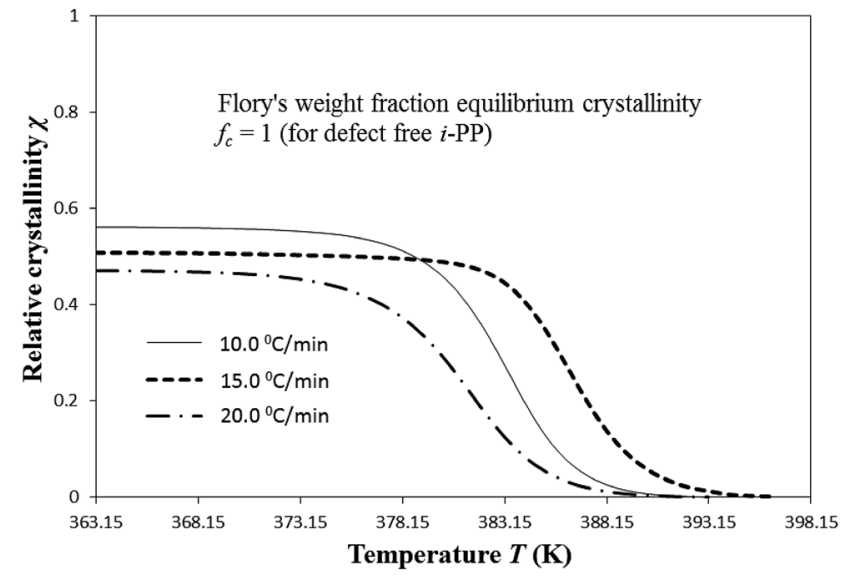

Fig. 9 Variation of temperature-dependent instantaneous Cycle 2 crystallinity $\chi$ with cooling temperature.

(i) $\chi$ shows to be temperature-dependent; and

(ii) $\chi(T)$ increases as $\theta$ increases, and $D$ and critical stable crystallite sequence number $n^{*}$ decrease with the decrease in $T$.

The asymptotic value of $\chi$ equals to the Cycle 2 DSC \% crystallinity of the i-PP sample. $\chi$ (asymptotic or nonasymptotic), for a given cooling rate, is always much less than Flory's weight fraction equilibrium crystallinity $f_{\mathrm{c}}$, which for a defect-free i-PP homopolymer is equal to unity. The predicted crystallinity difference (with respect to $f_{\mathrm{c}}=1$ ) may be attributed to the topology and the eventual kinetic restraint with reference to Flory's equilibrium theory. ${ }^{16,61-64}$ Therefore, crystallinity may be improved by decreasing the kinetic and topological restraints. By topology, we mean the crystallizable isotactic polypropylene sequence length distribution SLD (due to stereodefects), the density of chain entanglement, and the configuration of the folding lamellae. Note that according to the equilibrium theory, only sequences exceeding a certain critical length crystallize. The SLD reduces the exceedingly long sequences required for equilibrium. On the other hand, the resulting kinetic constraint imposes hindrance to nucleation and crystal growth, and impinges the growing centers. The crystallization of particularly the very long sequences becomes especially difficult. Consequently, the experimental i-PP does not achieve the structural topology stipulated by the equilibrium requirements, and its melting point, heat of fusion, and crystallinity decrease.

Here, we address the effects of the level of undercooling $\theta$ and crystal surface free energy $D$ on the melting behavior of iPP. See Fig. 10. $\theta$ and $D$ were calculated using eqn (12) and (13), respectively, and the Cycle 3 DSC melting data. The lamellar thickness $L$ of folded chain crystal (FCC) of the experimental Z-N i-PP-a random stereo-copolymer ${ }^{40-42}$ with configurational defects along the backbone-was calculated using the following version of Gibbs-Thompson thermodynamic equation: ${ }^{19-23}$

$$
L=\frac{2 \sigma_{\mathrm{e}}}{\Delta H_{\mathrm{u}}}\left(\frac{T_{\mathrm{m}, \mathrm{i}-\mathrm{PP}}^{0}}{T_{\mathrm{m}, \mathrm{i}-\mathrm{PP}}^{0}-T_{\mathrm{m}}}\right)
$$



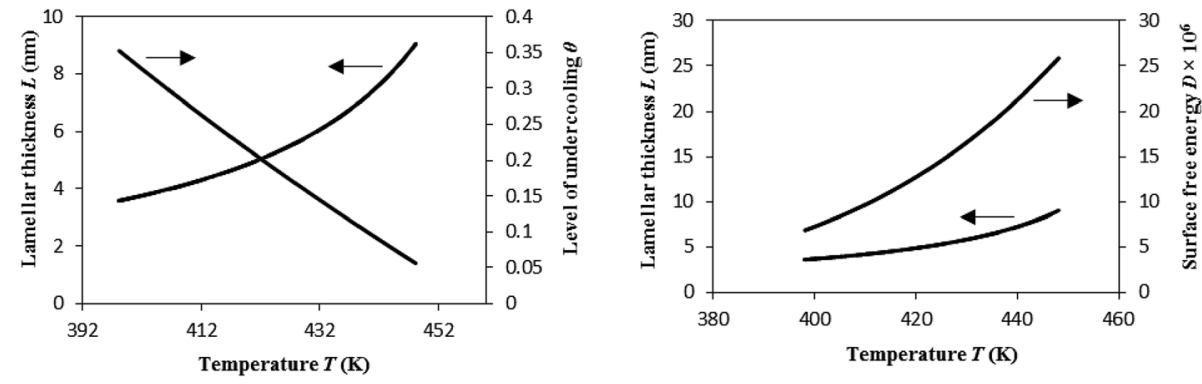

Fig. 10 Variation of lamellar thickness $L$, level of undercooling $\theta$, and crystal surface free energy $D$ as a function of cooling temperature. Plots for all the experimental heating rates overlapped.

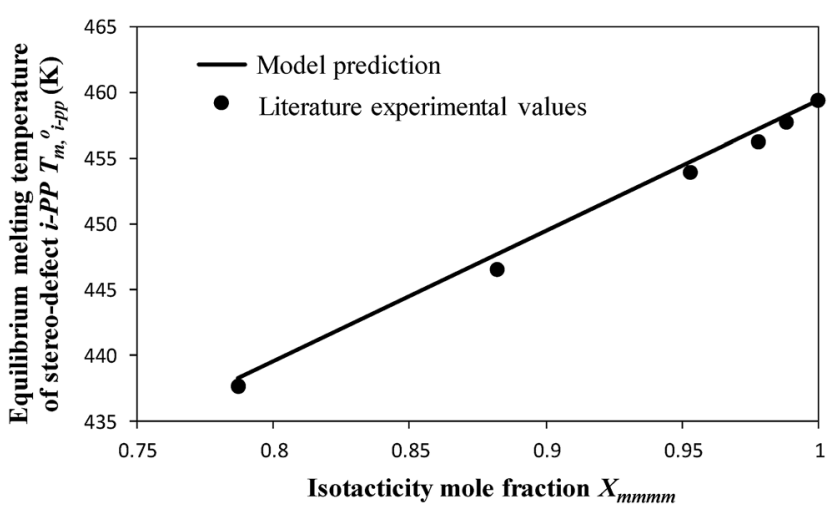

Fig. 11 Comparison of the thermodynamic model-predicted equilibrium melting temperature $T_{\mathrm{m}, \mathrm{i}-\mathrm{PP}}^{0}$ of an i-PP (with stereo-defects) with that published in the literature.

where $T_{\mathrm{m}, \mathrm{i}-\mathrm{PP}}^{0}$ is the equilibrium melting temperature of an i-PP with stereo-defects, and $T_{\mathrm{m}}$ is the DSC-measured Cycle 3 melting temperature. $\Delta H_{\mathrm{u}}$ and $\sigma_{\mathrm{e}}$ have been already defined in eqn (12) and (13), respectively. Eqn (21) corrects for the stereodefects in the i-PP backbone. Here, the i-PP pendant $-\mathrm{CH}_{3}-$ group, with that in the stereo-defect, is included in the folded lamella. ${ }^{18,38,42}$ Under such a situation, Cheng et al., ${ }^{42}$ using thermodynamic considerations, derived the following equation:

$$
T_{\mathrm{m}, \mathrm{i}-\mathrm{PP}}^{0}=T_{\mathrm{m}}^{0}\left[1-\frac{\left(1-X_{\mathrm{mmmm}}\right) \varepsilon_{0}}{\Delta H_{\mathrm{f}}^{0}}\right]
$$

where $\varepsilon_{0}\left(1.90 \mathrm{~kJ} \mathrm{~mol}^{-1}\right)$ is the excess energy of a perfect i-PP crystal. $X_{\mathrm{mmmm}}, T_{\mathrm{m}}^{0}$, and $\Delta H_{\mathrm{f}}^{0}$ have been already defined in eqn (11), (12), and (19), respectively. Fig. 11 shows that eqn (21) very closely matches the experimental data published in the literature. ${ }^{42,65}$ The standard deviation (STD), calculated by using eqn (22), is 1.33 .

$$
\mathrm{STD}=\sqrt{\frac{1}{N_{\text {data }}} \sum_{1}^{N_{\text {data }}}\left[T_{\mathrm{m}, \mathrm{i}, \mathrm{PP}}^{0}(\text { experimental })-T_{\mathrm{m}, \mathrm{-PP}}^{0}(\text { eqn }(20))\right]^{2}}
$$

The following alpha-phase perfect crystal (of infinite lamellar thickness and molar mass) parametric values ${ }^{16,30,31}$ were applied to the above equations: $T_{\mathrm{m}}^{0}=459.1 \mathrm{~K}, \Delta H_{\mathrm{f}}^{\circ}=8786.4 \mathrm{~J} \mathrm{~mol}^{-1}$ $\left(207 \mathrm{~J} \mathrm{~g}^{-1}\right)$, and $\sigma_{\mathrm{e}}($ crystal specific surface free energy $)=0.096 \mathrm{~J} \mathrm{~m}^{-2}$.

As per Flory's thermodynamic equilibrium theory, melting and crystallization are both reversible. This figure shows that melting first starts with the smaller defect-rich lamellae at lower temperatures, and higher undercooling favors this. On the other hand, the larger defect-free lamellae melt later at higher temperatures, which occurs due to lower level of undercoling $\theta$. In either case, the lamella melting temperature is always less than the equilibrium melting temperature $T_{\mathrm{m}}^{0}$ of the perfect crystalline i-PP (459.1 K). Compare Fig. 10 observation with that of Fig. 4. However, the effect of crystal surface free energy $D$ on melting behavior of i-PP differs from that of $\theta$. Here, $D$, unlike $\theta$, increases as the melting temperature increases, and consequently the smaller to larger lamellae sequentially melt. In
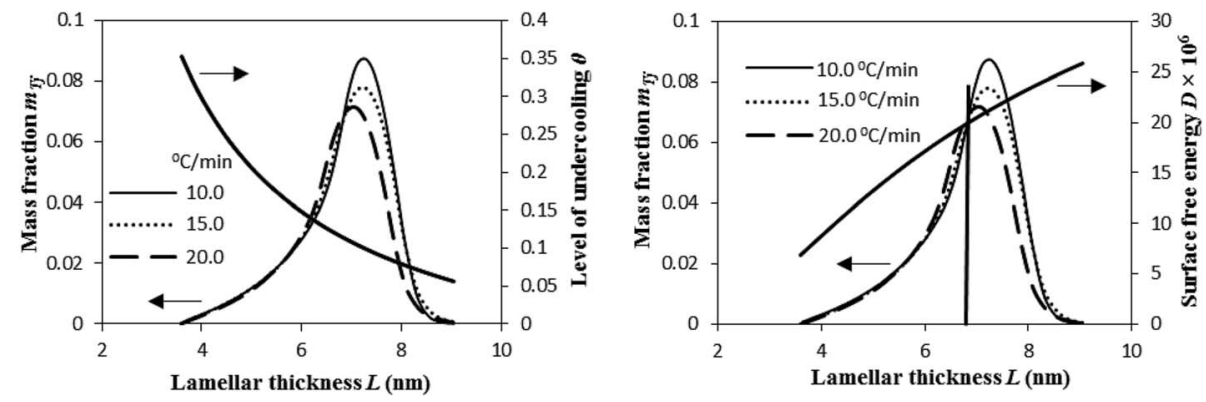

Fig. 12 Variation of mass fraction of crystal lamellae melted, level of undercooling $\theta$, and crystal surface free energy $D$ as a function of lamellar thickness $L$. 
summary, lamellar thickening occurs with the decrease of $\theta$, and increase of melting temperature and $D$. This phenomenon significantly increases in the molten mobile phase. The chain sliding diffusion theory proposed by Hikosaka et al., ${ }^{66-68}$ combined with the above variation of $\theta$ and $D$ support this lamellar thickening behavior. The heating rate does not affect this phenomenon. These are insightful findings, and to the best of our knowledge, they have not been reported earlier in the literature.

Overall, $\theta$ and $D$ inversely influence the i-PP melting phenomenon. This can be explained as follows. Eqn (12) shows that $\theta$ linearly increases as a function of $\frac{\Delta T}{T}\left(\right.$ where $\left.\Delta T=T_{\mathrm{m}}^{0}-T\right)$, and it mathematically expresses the undercooling profile of the experimental i-PP with reference to the polypropylene perfect crystal (having infinite MW and lamellar thickness, and $\theta=0$ ). Hence, it eventually shows the temperature gradient effect on i-PP melting. On the other hand, $D$ decreases exponentially as a function of non-dimensional crystal surface free energy $\frac{\sigma_{\mathrm{e}} a_{\mathrm{o}}}{R T}$. See eqn (13). The above variational trends of $\theta$ and $D$ are fundamentally related to the topologies of the lamellae and crystallite surface, which are affected by the architecture of the i-PP backbones. See Fig. 4 .

Fig. 12 illustrates that the lamellar thickness $L$ of the experimental i-PP crystal shows a distribution during melting. We calculated this lamellar thickness distribution (LTD) as follows. $L$ was estimated using eqn (20) and (21), and the corresponding mass fraction $m_{T_{j}}$ (at a given temperature $T_{j}$ ), by applying eqn (23) and the Cycle 3 DSC data: ${ }^{25}$

$$
m_{T_{j}}=\frac{\int_{T_{i}}^{T_{j}}\left(\frac{\mathrm{d} H}{\mathrm{~d} T}\right) \mathrm{d} T-\int_{T_{i}}^{T_{j-1}}\left(\frac{\mathrm{d} H}{\mathrm{~d} T}\right) \mathrm{d} T}{\int_{T_{i}}^{T_{\infty}}\left(\frac{\mathrm{d} H}{\mathrm{~d} T}\right) \mathrm{d} T}
$$

The above figure also demonstrates the relation that exists among the LTD, the level of undercooling $\theta$, and crystal surface free energy $D$. The LTD plots particularly show that a substantial mass fraction of the crystal lamellae melts at lower $\theta$ and higher $D$. See the LTD at the right side of the vertical line. Also, the most probable lamellar thickness $L_{\mathrm{MP}}$, melting temperature $T_{\mathrm{MP}}$, level of undercooling $\theta_{\mathrm{MP}}$, and crystal surface free energy $D_{\mathrm{MP}}$ are found to be a weak function of cooling rate $\beta$. See Table 1 . All these most probable properties, for a given $\beta$, that correspond to the maximum mass fraction of the LTD, show lower $\theta$ and higher $D$.

\section{Conclusions}

Isotactic polypropylene (i-PP) is a highly important polyolefin thermoplastic that fabricates multitude of end-products. Melting and crystallization are integral parts of this fabrication process. Therefore, this report revisits the nonisothermal crystallization kinetics and melt behavior of a commercial Ziegler-Natta i-PP by integrating a new nonisothermal crystallization model (which we published in 2013), Flory's thermodynamic equilibrium theory, Gibbs-Thompson equation, and nonisothermal DSC experiments. Flory's equilibrium theory has been applied by considering that an i-PP can be microstructurally defined to be a random stereo-copolymer having configurational defects along the backbone. By applying the above simple conceptual integration, the relative crystallinity $\alpha$, temperature-dependent instantaneous crystallinity $\chi$, the crystallization kinetic triplet, and lamellar thickness and melting temperature have been duly correlated, as appropriate, to the following dimensionless factors-level of undercooling $\theta$, crystal surface free energy $D$, and critical stable crystallite sequence number $n^{*}$. Consequently, new insightful results, interpretations, and explanations have been concluded. In particular, the following can be listed:

- The nonisothermal primary and secondary crystallizations of i-PP occur isokinetically with constant (temperature-, entropy-, and cooling rate-invariant) apparent kinetic energy $E_{\mathrm{a}}$, which is also unaffected by the level of undercooling $\theta$, crystal surface free energy $D$, and the lamella-inclusive pendant $-\mathrm{CH}_{3}$ group of the i-PP repeat unit. The crystal dimension $n_{\mathrm{d}}$ varies, independent of $\theta$ and $D$, from cylinder to sphere. Low and high system disorder (entropy), among other factors, favor the growth of spherulitic and cylindrical crystals, respectively. Instantaneous (athermal/heterogeneous) and sporadic (thermal/homogeneous) nucleation processes may co-occur. The cooling rate and system entropy influence the rate of nucleation over that of the crystal growth.

- The very rapid primary and slow secondary crystallizations originate from the increase and decrease in the level of undercooling $\theta$ and crystal surface free energy $D$, respectively. In primary crystallization, the $\mathrm{d} \alpha / \mathrm{d} \theta$ and $-\mathrm{d} \alpha / \mathrm{d} D$ are much higher than the corresponding derivatives in secondary crystallization. Despite having inverse characteristics, $\theta$ and $D$, do not change $E_{\text {a }}$, either as a function of cooling temperature $T$ or rate $\beta$.

- The temperature-dependent instantaneous (dynamic) crystallinity $\chi(T)$ increases as the level of undercooling $\theta$ increases, and crystal surface free energy $D$ decreases with the decrease in $T$ till an asymptotic value is reached.

- Smaller lamellae first melt at lower temperatures and higher level of undercooling $\theta$. The reverse applies to the larger lamellae. The overall lamella melting temperature is always below the equilibrium melting temperature i-PP (459.1 K). The crystal surface free energy $D$, unlike $\theta$, increases as the melting temperature increases, and consequently the smaller to larger lamellae sequentially melt. A substantial mass fraction of the lamella melts at lower $\theta$ and higher $D$.

- Lamellar thickening occurs with the decrease of $\theta$, and increase of melting temperature, and $D$. This phenomenon significantly increases in the molten mobile phase. The chain sliding diffusion theory proposed by Hikosaka et al., ${ }^{66-68}$ combined with the above variation of $\theta$ and $D$ support this lamellar thickening behavior.

The approach of this study also applies to evaluate the influence of catalyst structure, backbone defect types, and their distribution on the crystallization and melt behavior of i-PP.

\section{Conflicts of interest}

There are no conflicts to declare. 


\section{Acknowledgements}

The authors greatly acknowledge the financial support provided for this study by King Abdulaziz City for Science and Technology (KACST) via the Science and Technology Unit (STU) at King Fahd University of Petroleum \& Minerals (KFUPM) through Project Number 14-PET-283-04 as part of the National Science and Technology Innovation Plan (MAARIFAH). The technical assistance provided by the Department of Chemical Engineering, the Center for Refining \& Petrochemicals (CRP) at KFUPM, Dhahran, Saudi Arabia; and SABIC Technical Center at Riyadh is also gratefully acknowledged. The technical assistance of Messrs. Anwar Hossaen and Sarath P. Unnikari and the gift of the polypropylene sample by National Industrialization Company (Tasnee) are thankfully appreciated.

\section{References}

1 C. C. Hsu, P. H. Geil, H. Miyaji and K. Asai, J. Polym. Sci., Part B: Polym. Phys., 1986, 24, 2379-2401.

2 M. L. Di Lorenzo and C. Silvestre, Prog. Polym. Sci., 1999, 24, 917-950.

3 Y. Mubarak, E. M. A. Harkin-Jones, P. J. Martin and M. Ahmad, Polymer, 2001, 42, 3171-3182.

4 A. Gradys, P. Sajkiewicz, A. A. Minakov, S. Adamovsky, C. Schick, T. Hashimoto and K. Saijo, Mater. Sci. Eng., A, 2005, 413, 442-446.

5 S. Qiu, Y. Zheng, A. Zeng and Y. Guo, Thermochim. Acta, 2011, 512, 28-33.

6 M. Van Drongelen, T. B. Van Erp and G. W. M. Peters, Polymer, 2012, 53, 4758-4769.

7 S. Zhao and Z. Xin, J. Appl. Polym. Sci., 2009, 112, 1471-1480. 8 L. Xu, X. Zhang, K. Xu, S. Lin and M. Chen, Polym. Int., 2010, 59, 1441-1450.

9 J. Qin, X. Chen, J. Xu, Y. Wang, Y. Tian and S. Wu, J. Appl. Polym. Sci., 2010, 117, 1047-1054.

10 Y. H. Shi and Q. Dou, J. Therm. Anal. Calorim., 2013, 112, 901-911.

11 S. C. Tjong and S. A. Xu, Polym. Int., 1997, 44, 95-103.

12 W. Xu, M. Ge and P. He, J. Polym. Sci., Part B: Polym. Phys., 2002, 40, 408-414.

13 A. Fereidoon, M. G. Ahangari and S. Saedodin, J. Macromol. Sci., Part B: Phys., 2009, 48, 25-40.

14 M. G. Ahangari, A. Fereidoon, M. Kordani and H. Garmabi, Polym. Bull., 2011, 66, 239-258.

15 M. Atiqullah, M. M. Hossain, M. S. Kamal, M. A. Al-Harthi, M. J. Khan, A. Hossaen and I. Hussain, AIChE J., 2013, 59, 200-212.

16 C. Ruiz-Orta, J. P. Fernandez-Blazquez, A. M. Anderson-Wile, G. W. Coates and R. G. Alamo, Macromolecules, 2011, 44, 3436-3451.

17 P. J. Flory, Trans. Faraday Soc., 1955, 51, 848-857.

18 B. Crist and P. R. Howard, Macromolecules, 1999, 32, 30573067.

19 S. Hosoda, Y. Nozue, Y. Kawashima, K. Suita, S. Seno, T. Nagamatsu, K. B. Wagener, B. Inci, F. Zuluaga, G. Rojas and J. K. Leonard, Macromolecules, 2011, 44, 313-319.
20 P. J. Flory, J. Chem. Phys., 1949, 17, 223-240.

21 F. Chen, R. A. Shanks and G. Amarasinghe, Polym. Int., 2004, 53, 1795-1805.

22 M. R. Kamal, L. Feng and T. Huang, Can. J. Chem. Eng., 2002, 80, 432-442.

23 L. Feng and M. R. Kamal, Can. J. Chem. Eng., 2004, 82, 12391251.

24 M. Atiqullah, S. Anantawaraskul, A. M. Emwas, M. A. AlHarthi, I. Hussain, A. Ul-Hamid and A. Hossaen, Ind. Eng. Chem. Res., 2013, 52, 9359-9373.

25 M. Atiqullah, M. M. Hossain, S. Adamu and A. Hossaen, Polym. Int., 2014, 63, 1824-1834.

26 M. Atiqullah, S. Adamu, M. M. Hossain, M. A. Al-Harthi, S. Anantawaraskul and A. Hossaen, J. Taiwan Inst. Chem. Eng., 2014, 45, 1982-1991.

27 C. Grein, M. Gahleitner, B. Knogler and S. Nestelberger, Rheol. Acta, 2007, 46, 1083-1089.

28 M. Atiqullah, M. S. Winston, J. E. Bercaw, I. Hussain, A. Fazal, M. A. Al-Harthi, A. H. Emwas, M. J. Khan and A. Hossaen, Polym. Degrad. Stab., 2012, 97, 1164-1177.

29 G. D. Wignall, R. G. Alamo, J. D. Londono, L. Mandelkern, M. H. Kim, J. S. Lin and G. M. Brown, Macromolecules, 2000, 33, 551-561.

30 G. W. Ehrenstein and R. P. Theriault, Polymeric Materials: Structure, Properties, and Applications, Hanser Verlag, 2001, pp. 67-78.

31 D. W. Van der Meer, Structure-property Relationship in Isotactic Polypropylene, PhD Thesis, University of Twente, The Netherlands, 2003.

32 M. Atiqullah, M. A. Al-Harthi, S. Anantawaraskul and A. H. M. Emwas, J. Chem. Sci., 2015, 127, 717-728.

33 T. Hayashi, Y. Inoue, R. Chûjô and T. Asakura, Polymer, 1988, 29, 138-143.

34 R. Paukkeri, T. Väänänen and A. Lehtinen, Polymer, 1993, 34, 2488-2494.

35 M. Avrami, J. Chem. Phys., 1939, 7, 1103-1112.

36 A. T. Lorenzo, M. L. Arnal, J. Albuerne and A. J. Müller, Polym. Test., 2007, 26, 222-231.

37 M. Muthukumar, Adv. Chem. Phys., 2004, 128, 1-64.

38 D. L. Vanderhart, M. R. Nyden, R. G. Alamo and L. Mandelkern, Polym. Prepr., 2000, 82, 140-142.

39 V. Busico, Adv. Polym. Sci., 2013, 257, 37-58.

40 D. W. Meer, J. Varga and G. J. Vancso, eXPRESS Polym. Lett., 2015, 9, 233-254.

41 J. R. Severn and J. C. Chadwick, Tailor-made Polymers: Via Immobilization of Alpha-Olefin Polymerization Catalysts, Wiley-VCH Verlag GmbH \& Co. KGaA, Weinheim, Germany, 2008.

42 S. Z. D. Cheng, J. J. Janimak, A. Zhang and E. T. Hsieh, Polymer, 1991, 32, 648-655.

43 G. Allegra, R. Marchessault and S. Bloembergen, J. Polym. Sci., Part B: Polym. Phys., 1992, 30, 809-815.

44 I. S. Gradshteyn and I. M. Ryzhik, Table of Integrals, Series, and Products, Academic Press, New York, 4th edn, 1980, p. 93.

45 G. Z. Papageorgiou, D. S. Achilias and G. P. Karayannidis, Polymer, 2010, 51, 2565-2575. 
46 I. Acar, A. Durmuş and S. Özgümüss, J. Appl. Polym. Sci., 2007, 106, 4180-4191.

47 D. Olmos, C. Dominguez, P. D. Castrillo and J. GonzalezBenito, Polymer, 2009, 50, 1732-1742.

48 A. Adhikari and K. Lozano, J. Polym. Res., 2011, 18, 875-880.

49 A. Hammami, J. E. Spruiell and A. K. Mehrotra, Polym. Eng. Sci., 1995, 35, 797-804.

50 P. Supaphol, N. Dangseeyun, P. Srimoaon and M. Nithitanakul, Thermochim. Acta, 2003, 406, 207-220.

51 K. A. Jackson, Kinetic Processes: Crystal Growth, Diffusion, and Phase Transitions in Materials, Wiley-VCH, Weinheim, Germany, 2nd edn, 2012.

52 S. S. Sahay and K. Krishnan, Thermochim. Acta, 2005, 430, 23-29.

53 B. Monrabal and P. del Hierro, Anal. Bioanal. Chem., 2011, 399, 1557-1561.

54 J. C. Randall, Polymer Sequence Determination: Carbon 13 NMR Method, Academic Press, New York, 1977.

55 A. K. Galwey, Eradicating Erroneous Arrhenius Arithmetic, Thermochim. Acta, 2003, 399, 1-29.

56 A. K. Galwey, Thermochim. Acta, 2003, 397, 249-268.

57 K. Chrissafis, K. M. Paraskevopoulos, S. Y. Stavrev, A. Docoslis, A. Vassiliou and D. N. Bikiaris, Thermochim. Acta, 2007, 465, 6-17.
58 G. Papageorgiou, D. N. Bikiaris and K. Chrissafis, Polym. Int., 2010, 59, 1630-1638.

59 H. Eloussifi, J. Farjas, P. Roura and M. Dammak, J. Therm. Anal. Calorim., 2012, 108, 597-603.

60 Q. Zhou, A. Wang, H. Li, Z. Luo, T. Zheng, L. Zhang and Y. Hu, RSC Adv., 2016, 6, 75023-75031.

61 R. Alamo, R. Domszy and L. Mandelkern, J. Phys. Chem., 1984, 88, 6587-6595.

62 R. Alamo and L. Mandelkern, Macromolecules, 1991, 24, 6480-6493.

63 R. Alamo and L. Mandelkern, Thermochim. Acta, 1994, 238, 155-201.

64 B. Crist and T. M. Finerman, Polymer, 2005, 46, 8745-8751.

65 K. Yamada, M. Hikosaka, A. Toda, S. Yamazaki and K. Tagashira, Macromolecules, 2003, 36, 4790-4801.

66 M. Hikosaka, S. Rastogi, A. Keller and H. Kawabata, J. Macromol. Sci., Part B: Phys., 1992, 31, 87-131.

67 S. Rastogi, M. Hikosaka, H. Kawabata and A. Keller, Macromolecules, 1991, 24, 6384-6391.

68 M. Hikosaka, H. Okada, A. Toda, S. Rastogi and A. Keller, J. Chem. Soc., Faraday Trans., 1995, 91, 2573-2579. 